\title{
Synthesis of $\alpha-C_{2}$-mannosides and their conversion to fluorinated pseudo-glycopeptides
}

\author{
Florent Poulain, Anne-Lise Serre, Jérôme Lalot, Eric Leclerc, Jean-Charles Quirion \\ Laboratoire d'Hétérochimie Organique, UMR 6014 CNRS, IRCOF, INSA et Université de Rouen, 1 rue Tesnière, \\ 76821 Mont Saint-Aignan Cedex, France \\ eric.leclerc@insa-rouen.fr
}

I. Addition of difluoroketene silyl acetal 4 to 3,4,6-tri-O-acetyl-D-glucal 5 .........................S3

Ethyltrimethylsilyl-2,2-difluoroketene acetal (4).................................................................................. S3

Ethyl 2-(4,6-Di-O-acetyl-2,3-dideoxy-D-erythro-hex-2-enopyranosyl)-2,2-difluoroacetate (6)...................S3

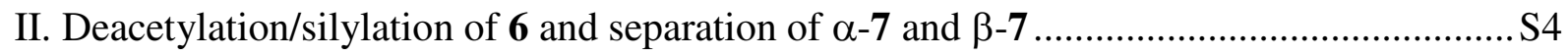

Ethyl 2-(6-O-(tert-butyldiphenylsilyl)-2,3-dideoxy- $\alpha$-D-erythro-hex-2-enopyranosyl)-2,2-difluoroacetate $(\alpha-7)$.

Ethyl 2-(6-O-(tert-butyldiphenylsilyl)-2,3-dideoxy- $\beta$-D-erythro-hex-2-enopyranosyl)-2,2-difluoroacetate $(\beta-7)$.

III. Dihydroxylation reactions S5

Ethyl 2-(6-O-(tert-butyldiphenylsilyl)- $\beta$-D-allopyranosyl)-2,2-difluoroacetate (8). S5

Ethyl 2-(6-O-(tert-butyldiphenylsilyl)- $\alpha$-D-mannopyranosyl)-2,2-difluoroacetate (9). S6

IV. Synthesis of pseudo-glycopeptides S7

Ethyl 2-(2,3,4-tri-O-benzyl-6-O-(tert-butyldiphenylsilyl)- $\alpha$-D-mannopyranosyl)-2,2-diflu-oroacetate (12).

Di-O-benzyl $\quad N$-[2-(2,3,4-tri-O-benzyl-6-O-(tert-butyldiphenylsilyl)- $\alpha$-D-mannopyranosyl)-2,2-difluoroacetyl]-L-glutamate (13). S8

$N$-(2,2-Difluoro-2-( $\alpha$-D-mannopyranosyl)acetyl)-L-glutamic acid $(\alpha-3 \boldsymbol{a})$.

Ethyl N-[2-(2,3,4-tri-O-benzyl-6-O-(tert-butyldiphenylsilyl)- $\alpha$-D-mannopyranosyl)-2,2-difluoroacetyl]-3aminobenzoate (14). S9

$N$-[2,2-Difluoro-2-( $\alpha$-D-mannopyranosyl)acetyl]-3-aminobenzoic acid $(\alpha-3 \boldsymbol{b})$. S10 
General Methods. All reactions were carried out under an argon or nitrogen atmosphere with dry solvents under anhydrous conditions, unless otherwise noted. Dry THF, 1,4-dioxane, $\mathrm{MeCN}$ or $\mathrm{CH}_{2} \mathrm{Cl}_{2}$ were obtained by drying over $\mathrm{Na}$ /benzophenone (THF and 1,4dioxane) or $\mathrm{CaH}_{2}(\mathrm{MeCN})$ or $\mathrm{P}_{2} \mathrm{O}_{5}\left(\mathrm{CH}_{2} \mathrm{Cl}_{2}\right)$ and distillation. All reagents were purchased from commercial sources and used without further purification. Reactions were monitored by thin-layer chromatography (TLC) carried out on $0.25 \mathrm{~mm}$ silica gel plates using UV light as a visualizing agent and an ethanolic solution of phosphomolybdic acid or vanilline, and heat as developing agents. Silica gel (60, particle size $0.040-0.063 \mathrm{~mm}$ or $0.070-0.200 \mathrm{~mm}$ ) was used for flash column chromatography. ${ }^{1} \mathrm{H}$ NMR, ${ }^{13} \mathrm{C}$ NMR and ${ }^{19} \mathrm{~F}$ NMR spectra were recorded on a $300 \mathrm{MHz}$ instrument and calibrated using residual undeuterated solvent as an internal reference and $\mathrm{CFCl}_{3}$ as external reference. The COSY and HOESY experiments for compound 9 were recorded on a $400 \mathrm{MHz}$ instrument. The following abbreviations were used to explain the multiplicities: $\mathrm{s}=$ singlet, $\mathrm{d}=$ doublet, $\mathrm{t}=$ triplet, $\mathrm{m}=$ multiplet, app $=$ apparent . Mass spectrometry (MS) experiments were performed using electrospray ionization (ESI), electronic impact $(\mathrm{EI}-70 \mathrm{eV})$ or chemical ionization $(\mathrm{CI} / \mathrm{DCI}-200 \mathrm{eV})$. Optical rotations were measured at $20^{\circ} \mathrm{C}$ and with $\lambda=589 \mathrm{~nm}$; concentrations are expressed in cg. $\mathrm{mL}^{-1}$. 


\section{Addition of difluoroketene silyl acetal 4 to 3,4,6-tri-O-acetyl-D-glucal 5}<smiles>O=C([O-])C(F)(F)Br</smiles>

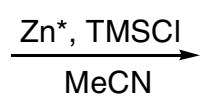<smiles>CCOC(O[SiH3])=C(F)F</smiles>

Ethyltrimethylsilyl-2,2-difluoroketene acetal (4). Zinc dust (3 g, 46mmol) in a Schlenk tube was dried by heating under vacuum and cooled to room temperature under argon atmosphere. Acetonitrile $(16 \mathrm{~mL})$ was added and the zinc was activated under vigorous stirring by adding dibromoethane $(3 \% \mathrm{~mol})$ and $\mathrm{TMSCl}(5 \% \mathrm{~mol})$. The mixture was cooled to room temperature and a solution of ethyl bromodifluoroacetate $(3.75 \mathrm{~mL}, 27 \mathrm{mmol})$ in acetonitrile $(6 \mathrm{~mL})$ was added dropwise over 1 hour. The mixture was then stirred for one more hour after the end of the addition and $\mathrm{TMSCl}(5 \mathrm{~mL}, 40 \mathrm{mmol})$ was then added. After one hour, the reaction mixture was then cooled to $4{ }^{\circ} \mathrm{C}$ and extracted with pentane $(8 \mathrm{~mL}+3 \times 4 \mathrm{~mL})$ under argon atmosphere. Care should be taken to avoid moisture contact with $\mathbf{4}$ which is used as a pentane solution without further purification.

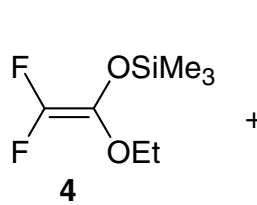

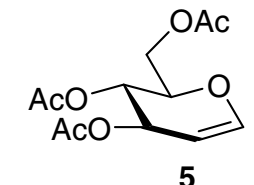

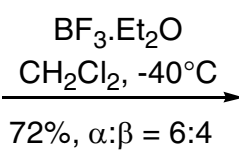

$72 \%, \alpha: \beta=6: 4$

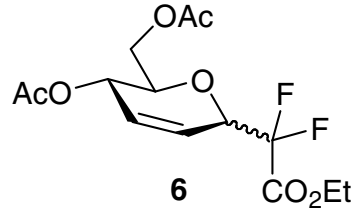

$\mathrm{C}_{14} \mathrm{H}_{18} \mathrm{~F}_{2} \mathrm{O}_{7}$

Mol. Wt.: 336.3

Ethyl 2-(4,6-Di-O-acetyl-2,3-dideoxy-D-erythro-hex-2-enopyranosyl)-2,2-difluoroacetate (6). To a solution of 3,4,6-tri- $O$-acetyl-D-glucal ${ }^{1} 5(2 \mathrm{~g}, 7.35 \mathrm{mmol})$ in dichloromethane (22 $\mathrm{mL})$ at $-40^{\circ} \mathrm{C}$ was added a solution of $4(24 \mathrm{mmol})$ in pentane and boron trifluoride diethyl etherate $(1 \mathrm{~mL}, 7.9 \mathrm{mmol})$. The solution was stirred at this temperature for $40 \mathrm{~min}$ and neutralized by adding a saturated aqueous $\mathrm{NaHCO}_{3}(15 \mathrm{~mL})$ and water $(15 \mathrm{~mL})$. The organic layer was separated and the aqueous phase extracted with ether $(2 \times 15 \mathrm{~mL})$. The combined organic extracts were washed with brine $(20 \mathrm{~mL})$, dried over magnesium sulphate and concentrated under reduced pressure. Purification by column chromatography over silica gel (30\% ethyl acetate in hexane) afforded $\mathbf{6}$ as a colourless oil and as a mixture of anomers (1.78

\footnotetext{
${ }^{1}$ Koreeda, M.; Shull, B. K.; Wu, Z. J. Carbohydrate Chem. 1996, 15, 955.
} 
g, 72\%). Fractions of reasonably pure $\alpha$ anomer can however be isolated: $\mathrm{Rf}=0.23$ (30\% EtOAc in cyclohexane). ${ }^{1} \mathrm{H}$ NMR $\left(300 \mathrm{MHz}, \mathrm{CDCl}_{3}\right) \delta 6.10(\mathrm{dt}, J=10.5 \mathrm{~Hz}, J=2.2 \mathrm{~Hz}, 1 \mathrm{H})$, 6.01-5.94 (m, 1H), 5.24-5.19 (m, 1H), 4.74-4.61 (m, 1H), $4.36(\mathrm{q}, J=7.2 \mathrm{~Hz}, 2 \mathrm{H}), 4.22(\mathrm{dd}$, $J=12.1 \mathrm{~Hz}, J=5.6 \mathrm{~Hz}), 4.11(\mathrm{dd}, J=12.1 \mathrm{~Hz}, J=2.7 \mathrm{~Hz}), 4.05-3.98(\mathrm{~m}, 1 \mathrm{H}), 2.08(\mathrm{~s}, 3 \mathrm{H})$, $2.06(\mathrm{~s}, 3 \mathrm{H}), 1.34(\mathrm{t}, J=7.2 \mathrm{~Hz}, 3 \mathrm{H}) .{ }^{19} \mathrm{~F} \mathrm{NMR}\left(282.5 \mathrm{MHz}, \mathrm{CDCl}_{3}\right) \delta-110.5(\mathrm{~d}, J=260.7 \mathrm{~Hz}$, $1 \mathrm{~F}),-117.7(\mathrm{dd}, J=260.7 \mathrm{~Hz}, J=22.6 \mathrm{~Hz}, 1 \mathrm{~F}) .{ }^{13} \mathrm{C} \mathrm{NMR}\left(75.5 \mathrm{MHz}, \mathrm{CDCl}_{3}\right) \delta 170.8,170.3$, $163.1(\mathrm{t}, J=30.2 \mathrm{~Hz}), 114.1(\mathrm{t}, J=254.7 \mathrm{~Hz}), 130.0,122.3,71.9(\mathrm{dd}, J=30.5 \mathrm{~Hz}, J=24.2$ $\mathrm{Hz}), 71.2,64.0,63.3,62.5,21.0,20.8,14.0$. The following analytical data were obtained from the mixture of anomers : IR (neat) $v_{\max } 2987,1764,1742 \mathrm{~cm}^{-1}$. MS (ESI+) $: \mathrm{m} / \mathrm{z}=359.0$ $\left([\mathrm{M}+\mathrm{Na}]^{+}\right)$. Anal. Calcd. for $\mathrm{C}_{14} \mathrm{H}_{18} \mathrm{~F}_{2} \mathrm{O}_{7}: \mathrm{C}$, 50.04; H, 5.40. Found C, 50.05; H, 5.22.

\section{Deacetylation/silylation of 6 and separation of $\alpha-7$ and $\beta-7$}

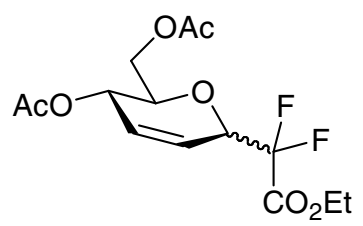

6

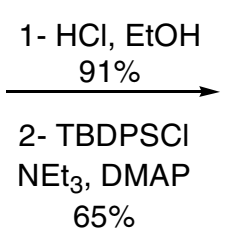

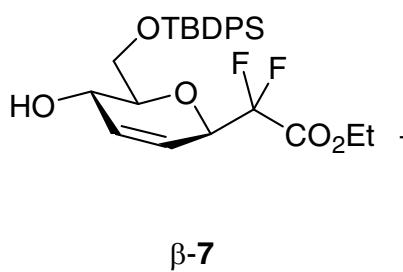

$\mathrm{C}_{26} \mathrm{H}_{32} \mathrm{~F}_{2} \mathrm{O}_{5} \mathrm{Si}$

Mol. Wt.: 490.6

Acetyl chloride ( $2 \mathrm{~mL}, 28 \mathrm{mmol})$ was added to a solution of $6(1.22 \mathrm{~g}, 3.63 \mathrm{mmol})$ in ethanol $(80 \mathrm{~mL})$ at room temperature. The reaction was monitored by TLC. After all starting material was consumed $(48 \mathrm{~h})$, the reaction mixture was neutralized with $\mathrm{NaHCO}_{3}(\sim 3.5 \mathrm{~g})$ and concentrated under reduced pressure. AcOEt $(60 \mathrm{~mL})$ was added to the residue and salts were removed by filtration. The organic solution was dried over magnesium sulfate and concentrated under reduced pressure to afford the expected diol $(0.85 \mathrm{~g}, 91 \%)$ which was used without further purification. To a solution of this crude compound $(1.1 \mathrm{~g}, 4.08 \mathrm{mmol})$ in $\mathrm{CH}_{2} \mathrm{Cl}_{2}(20 \mathrm{~mL})$ was added TBDPSCl $(1.4 \mathrm{~mL}, 5.4 \mathrm{mmol}), \mathrm{NEt}_{3}(1.5 \mathrm{~mL}, 10 \mathrm{mmol})$ and DMAP (60 mg $0.4 \mathrm{mmol}$ ). This solution was stirred for $24 \mathrm{~h}$ (consumption of the starting material was monitored by TLC) after which sat. $\mathrm{NaHSO}_{4}(15 \mathrm{~mL})$ and water $(15 \mathrm{~mL})$ were added. The mixture was extracted with $\mathrm{Et}_{2} \mathrm{O}(20 \mathrm{~mL}+2 \times 10 \mathrm{~mL})$ the combined organic layers were washed with brine $(30 \mathrm{~mL})$, dried over $\mathrm{MgSO}_{4}$ and concentrated under reduced pressure. Purification by column chromatography over silica gel (10\% EtOAc in cyclohexane) allowed the separation of $\alpha-7$ and $\beta-7$ (665 mg of $\alpha-7$ and $632 \mathrm{mg}$ of $\beta-7,65 \%$ global yield). 
Ethyl 2-(6-O-(tert-butyldiphenylsilyl)-2,3-dideoxy- $\alpha$-D-erythro-hex-2-enopyranosyl)-2,2difluoroacetate $(\alpha-7)$. $\mathrm{Rf}=0.56\left(30 \%\right.$ EtOAc in cyclohexane). $[\alpha]_{\mathrm{D}}:-9.6\left(c 0.55, \mathrm{CHCl}_{3}\right)$. ${ }^{1} \mathrm{H}$ NMR (300 MHz, $\left.\mathrm{CDCl}_{3}\right) \delta$ 7.71-7.64 (m, 4H), 7.49-7.35 (m, 6H), $6.17(\mathrm{dt}, J=10.5 \mathrm{~Hz}, J$ $=2.3 \mathrm{~Hz}, 1 \mathrm{H}), 5.85\left(\mathrm{bd}, J_{\mathrm{app}}=10.5 \mathrm{~Hz}, 1 \mathrm{H}\right), 4.58(\mathrm{dh}, J=21.6 \mathrm{~Hz}, J=2.5 \mathrm{~Hz}, 1 \mathrm{H}), 4.29-4.21$ $(\mathrm{m}, 1 \mathrm{H}), 4.25(\mathrm{q}, J=7.2 \mathrm{~Hz}, 2 \mathrm{H}), 3.92-3.83(\mathrm{~m}, 1 \mathrm{H}), 3.80-3.69(\mathrm{~m}, 2 \mathrm{H}), 2.74(\mathrm{~d}, J=4.7 \mathrm{~Hz}$, $1 \mathrm{H}), 1.23(\mathrm{t}, J=7.2 \mathrm{~Hz}, 3 \mathrm{H}), 1.06(\mathrm{~s}, 9 \mathrm{H}) .{ }^{19} \mathrm{~F} \mathrm{NMR}\left(282.5 \mathrm{MHz}, \mathrm{CDCl}_{3}\right) \delta-110.6(\mathrm{dd}, J=$ $261.6 \mathrm{~Hz}, J=4.6 \mathrm{~Hz}, 1 \mathrm{~F}),-118.4(\mathrm{dd}, J=261.6 \mathrm{~Hz}, J=21.8 \mathrm{~Hz}, 1 \mathrm{~F}) .{ }^{13} \mathrm{C} \mathrm{NMR}(75.5 \mathrm{MHz}$, $\left.\mathrm{CDCl}_{3}\right) \delta 163.2(\mathrm{dd}, J=32.8 \mathrm{~Hz}, J=29.3 \mathrm{~Hz}), 135.7,135.6,133.7,132.8,132.7,130.1,130.0$, 128.0, 127.9, 120.1, $114.3(\mathrm{dd}, J=263.3 \mathrm{~Hz}, J=255.3 \mathrm{~Hz}), 74.4(\mathrm{~d}, J=2.3 \mathrm{~Hz}), 71.6(\mathrm{dd}, J=$ $29.6 \mathrm{~Hz}, J=24.5 \mathrm{~Hz}), 65.2,65.1,63.1,26.9,19.3,14.0$. IR (neat) $v_{\max } 3435,3071,2931$, 2858, 1761. MS (ESI+) $: \mathrm{m} / \mathrm{z}=513.5\left([\mathrm{M}+\mathrm{Na}]^{+}\right)$. Anal. Calcd. for $\mathrm{C}_{26} \mathrm{H}_{32} \mathrm{~F}_{2} \mathrm{O}_{5} \mathrm{Si}: \mathrm{C}, 63.65 ; \mathrm{H}$, 6.57. Found C, 63.59; H, 6.64.

Ethyl 2-(6-O-(tert-butyldiphenylsilyl)-2,3-dideoxy- $\beta$-D-erythro-hex-2-enopyranosyl)-2,2difluoroacetate $(\beta-7) . \mathrm{Rf}=0.41\left(30 \%\right.$ EtOAc in cyclohexane). $[\alpha]_{\mathrm{D}}:+13.5\left(c 0.6, \mathrm{CHCl}_{3}\right) .{ }^{1} \mathrm{H}$ NMR $\left(300 \mathrm{MHz}, \mathrm{CDCl}_{3}\right) \delta$ 7.67-7.64 (m, 4H), 7.48-7.37 (m, 6H), $6.08\left(\mathrm{bd}, J_{\text {app }}=10.4 \mathrm{~Hz}\right.$, $1 \mathrm{H}), 5.86\left(\mathrm{bd}, J_{\text {app }}=10.4 \mathrm{~Hz}, 1 \mathrm{H}\right), 4.69-4.60(\mathrm{~m}, 1 \mathrm{H}), 4.34-4.21(\mathrm{~m}, 3 \mathrm{H}), 3.93(\mathrm{dd}, J=10.4$ $\mathrm{Hz}, J=5.1 \mathrm{~Hz}, 1 \mathrm{H}), 3.81(\mathrm{dd}, J=10.4 \mathrm{~Hz}, J=6.8 \mathrm{~Hz}, 1 \mathrm{H}), 3.55-3.48(\mathrm{~m}, 1 \mathrm{H}), 2.84(\mathrm{~d}, J=$ $3.7 \mathrm{~Hz}, 1 \mathrm{H}), 1.25(\mathrm{t}, J=7.1 \mathrm{~Hz}, 3 \mathrm{H}), 1.06(\mathrm{~s}, 9 \mathrm{H}) .{ }^{19} \mathrm{~F}$ NMR $\left(282.5 \mathrm{MHz}, \mathrm{CDCl}_{3}\right) \delta-114.0$ $(\mathrm{dd}, J=262.8 \mathrm{~Hz}, J=6.9 \mathrm{~Hz}, 1 \mathrm{~F}),-121.6(\mathrm{dd}, J=262.8 \mathrm{~Hz}, J=14.9 \mathrm{~Hz}, 1 \mathrm{~F}) .{ }^{13} \mathrm{C} \mathrm{NMR}(75.5$ $\left.\mathrm{MHz} \mathrm{CDCl}_{3}\right) \delta 162.7(\mathrm{dd}, J=32.8 \mathrm{~Hz}, J=30.5 \mathrm{~Hz}), 135.7,135.6,133.3,132.6,132.5,130.1$, 128.0, 121.6, $113.2(\mathrm{dd}, J=258.2 \mathrm{~Hz}, J=250.7 \mathrm{~Hz}), 77.4,74.5(\mathrm{dd}, J=30.5 \mathrm{~Hz}, J=26.4 \mathrm{~Hz})$, 65.9, 65.6, 63.1, 26.9, 19.2, 14.0. IR (neat) $v_{\max } 3436,2932,2858,1775$. MS (ESI+) $: \mathrm{m} / \mathrm{z}=$ 513.6 ([M+Na $\left.]^{+}\right)$. Anal. Calcd. for $\mathrm{C}_{26} \mathrm{H}_{32} \mathrm{~F}_{2} \mathrm{O}_{5} \mathrm{Si}: \mathrm{C}, 63.65 ; \mathrm{H}, 6.57$. Found C, 63.49; H, 6.61.

\section{Dihydroxylation reactions}

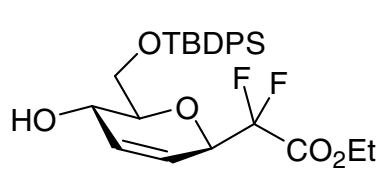

$\beta-7$

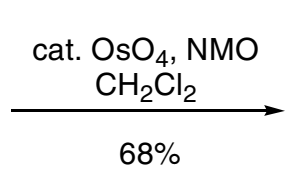

$68 \%$

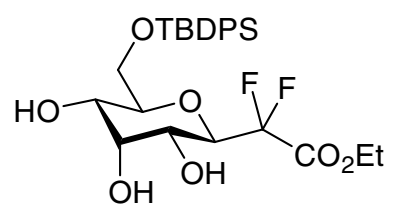

8
$\mathrm{C}_{26} \mathrm{H}_{34} \mathrm{~F}_{2} \mathrm{O}_{7} \mathrm{Si}$ Mol. Wt.: 524.6

Ethyl 2-(6-O-(tert-butyldiphenylsilyl)- $\beta$-D-allopyranosyl)-2,2-difluoroacetate (8). To a vigorously stirred solution of $\alpha-7(110 \mathrm{mg}, 0.22 \mathrm{mmol})$ in $\mathrm{CH}_{2} \mathrm{Cl}_{2}(2 \mathrm{~mL})$ was added $\mathrm{NMO}$ 
(120 mg, $1 \mathrm{mmol}$ ), few drops of water and a solution of osmium tetroxide (70 $\mu \mathrm{L}, 4 \%$ in water, $0.01 \mathrm{mmol}$ ). The reaction was monitored by TLC (reaction time $\sim 60 \mathrm{~h}$ ) and, after complete consumption of the starting material, the emulsion was dried over magnesium sulphate and directly transferred over a silica gel packed column for purification ( 0 to $1 \%$ $\mathrm{MeOH}$ in $\left.\mathrm{CH}_{2} \mathrm{Cl}_{2}\right)$ to afford 8 as a colourless oil $(79 \mathrm{mg}, 68 \%)$. $\mathrm{Rf}=0.18(50 \% \mathrm{EtOAc}$ in cyclohexane). $[\alpha]_{\mathrm{D}}:-1.3\left(c 0.48, \mathrm{CHCl}_{3}\right) .{ }^{1} \mathrm{H}$ NMR $\left(300 \mathrm{MHz}, \mathrm{CDCl}_{3}\right) \delta 7.70-7.64(\mathrm{~m}, 4 \mathrm{H})$, 7.48-7.37 (m, 6H), 4.31-4.09 (m, 4H), 3.98-3.68 (m, 5H), 3.47 (bs, 1H), 3.24 (bs, 1H), 2.86 (bs, 1H), $1.26(\mathrm{t}, J=7.1 \mathrm{~Hz}, 3 \mathrm{H}), 1.06(\mathrm{~s}, 9 \mathrm{H}) .{ }^{19} \mathrm{~F} \mathrm{NMR}\left(282.5 \mathrm{MHz}, \mathrm{CDCl}_{3}\right) \delta-117.4(\mathrm{dd}, J$ $=260.5 \mathrm{~Hz}, J=12.6 \mathrm{~Hz}, 1 \mathrm{~F}),-119.1(\mathrm{dd}, J=260.5 \mathrm{~Hz}, J=11.5 \mathrm{~Hz}, 1 \mathrm{~F}) .{ }^{13} \mathrm{C} \mathrm{NMR}(75.5$ $\left.\mathrm{MHz}, \mathrm{CDCl}_{3}\right) \delta 163.2(\mathrm{t}, J=30.5 \mathrm{~Hz}), 135.7,135.6,132.6,132.4,130.1,128.0,114.2(\mathrm{t}, J=$ 255.3 Hz), 74.0 (2C), 70.4, 69.9, 66.6 (d, $J=4.0 \mathrm{~Hz}), 65.2,63.1,26.9,19.3,14.0$. IR (neat) $v_{\max } 3380,3068,2936,2845,1769 \mathrm{~cm}^{-1}$. MS (ESI+) $: \mathrm{m} / \mathrm{z}=547.4\left([\mathrm{M}+\mathrm{Na}]^{+}\right)$. Anal. Calcd. for $\mathrm{C}_{26} \mathrm{H}_{34} \mathrm{~F}_{2} \mathrm{O}_{7} \mathrm{Si}: \mathrm{C}, 59.52 ; \mathrm{H}, 6.53$. Found C, 59.38; H, 6.76.
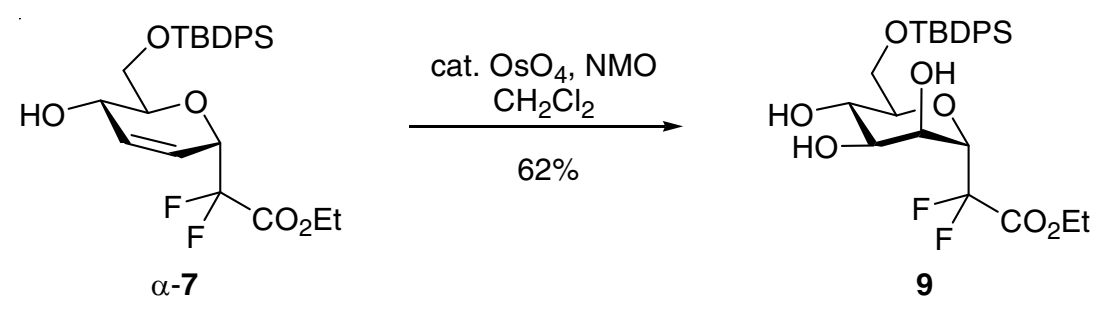

$\mathrm{C}_{26} \mathrm{H}_{34} \mathrm{~F}_{2} \mathrm{O}_{7} \mathrm{Si}$ Mol. Wt.: 524.6

Ethyl 2-(6-O-(tert-butyldiphenylsilyl)- $\alpha$-D-mannopyranosyl)-2,2-difluoroacetate (9). To a vigorously stirred solution of $\alpha-7(240 \mathrm{mg}, 0.49 \mathrm{mmol})$ in $\mathrm{CH}_{2} \mathrm{Cl}_{2}(3 \mathrm{~mL})$ was added NMO (250 mg, $2.1 \mathrm{mmol})$, few drops of water and a solution of osmium tetroxide (140 $\mu \mathrm{L}, 4 \%$ in water, $0.02 \mathrm{mmol}$ ). The reaction was monitored by TLC (reaction time $\sim 60 \mathrm{~h}$ ) and, after complete consumption of the starting material, the emulsion was dried over magnesium sulfate and directly transferred over a silica gel packed column for purification (0 to $10 \%$ $\mathrm{MeOH}$ in $\left.\mathrm{CH}_{2} \mathrm{Cl}_{2}\right)$ to afford 9 as colourless oil $(159 \mathrm{mg}, 62 \%)$. $[\alpha]_{\mathrm{D}}:+0.50\left(c 0.5, \mathrm{CHCl}_{3}\right) .{ }^{1} \mathrm{H}$ NMR $\left(300 \mathrm{MHz}, \mathrm{CDCl}_{3}\right) \delta 7.69-7.63(\mathrm{~m}, 4 \mathrm{H}), 7.48-7.36(\mathrm{~m}, 6 \mathrm{H}), 4.40(\mathrm{ddd}, J=27.1 \mathrm{~Hz}, J$ $=6.7 \mathrm{~Hz}, J=1.5 \mathrm{~Hz}, 1 \mathrm{H}), 4.31(\mathrm{bs}, 1 \mathrm{H}), 4.23(\mathrm{q}, J=6.9 \mathrm{~Hz}, 2 \mathrm{H}), 4.00-3.94(\mathrm{~m}, 2 \mathrm{H}), 3.90-$ $3.79(\mathrm{~m}, 2 \mathrm{H}), 3.79-3.74(\mathrm{~m}, 1 \mathrm{H}), 3.20(\mathrm{bs}, 1 \mathrm{H}), 3.10(\mathrm{bs}, 1 \mathrm{H}), 2.91(\mathrm{bs}, 1 \mathrm{H}), 1.21(\mathrm{t}, J=6.9$ $\mathrm{Hz}, 3 \mathrm{H}), 1.06$ (s, 9H). ${ }^{19} \mathrm{~F} \mathrm{NMR}\left(282.5 \mathrm{MHz}, \mathrm{CDCl}_{3}\right) \delta-108.5(\mathrm{dd}, J=258.6 \mathrm{~Hz}, J=6.4 \mathrm{~Hz}$, $1 \mathrm{~F}),-114.2(\mathrm{dd}, J=258.6 \mathrm{~Hz}, J=26.8 \mathrm{~Hz}, 1 \mathrm{~F}) .{ }^{13} \mathrm{C} \mathrm{NMR}\left(75.5 \mathrm{MHz}, \mathrm{CDCl}_{3}\right) \delta 162.8(\mathrm{dd}, J$ $=32.6 \mathrm{~Hz}, J=28.6 \mathrm{~Hz}), 135.7,132.6,130.2,128.0,115.2(\mathrm{t}, J=256.4 \mathrm{~Hz}), 77.0(\mathrm{dd}, J=29.6$ $\mathrm{Hz}, J=21.6 \mathrm{~Hz}), 75.0,71.8,69.9,66.2,65.5,63.4,26.9,19.3,14.0 . \mathrm{IR}$ (neat) $v_{\max } 3400,3072$, 
2932, 2858, $1771 \mathrm{~cm}^{-1}$. MS (ESI+) $: \mathrm{m} / \mathrm{z}=547.5\left([\mathrm{M}+\mathrm{Na}]^{+}\right)$. Anal. Calcd. for $\mathrm{C}_{26} \mathrm{H}_{34} \mathrm{~F}_{2} \mathrm{O}_{7} \mathrm{Si}$ : C, 59.52; H, 6.53. Found C, 59.32; H, 6.35.

\section{Synthesis of pseudo-glycopeptides}

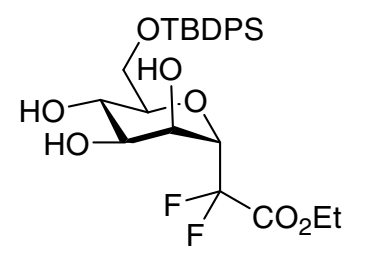

9

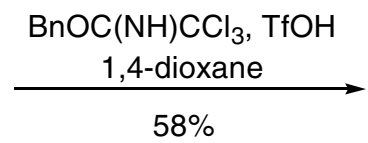

$58 \%$

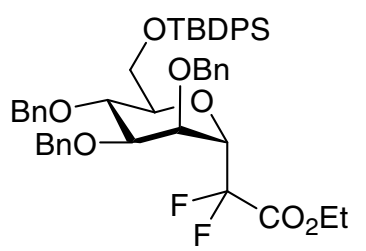

12
$\mathrm{C}_{47} \mathrm{H}_{52} \mathrm{~F}_{2} \mathrm{O}_{7} \mathrm{Si}$

Mol. Wt.: 795.0

\section{Ethyl 2-(2,3,4-tri-O-benzyl-6-O-(tert-butyldiphenylsilyl)- $\alpha$-D-mannopyranosyl)-2,2-diflu-} oroacetate (12). To a solution of $9(317 \mathrm{mg}, 0.60 \mathrm{mmol})$ in 1,4-dioxane $(6 \mathrm{~mL})$ was added molecular sieves, benzyltrichloroacetimidate $(1.68 \mathrm{~mL}, 9 \mathrm{mmol})$ and triflic acid (10 drops). The reaction was stirred overnight and quenched with sat. $\mathrm{NaHCO}_{3}(4 \mathrm{~mL})$ and water $(4 \mathrm{~mL})$. The mixture was extracted with EtOAc $(3 \times 6 \mathrm{~mL})$ and the combined organics were washed with brine $(8 \mathrm{~mL})$, dried over $\mathrm{MgSO}_{4}$ and concentrated under reduced pressure. Purification by column chromatography over silica gel (5\% EtOAc in cyclohexane) afforded $\mathbf{1 2}$ as a colourless oil (277 mg, 58\% yield). $\mathrm{Rf}=0.55$ (20\% EtOAc in cyclohexane). $[\alpha]_{\mathrm{D}}:+0.6(c 0.5$, $\left.\mathrm{CHCl}_{3}\right) .{ }^{1} \mathrm{H}$ NMR $\left(300 \mathrm{MHz}, \mathrm{CDCl}_{3}\right) \delta$ 7.69-7.62 (m, 4H), 7.44-7.20 (m, 21H), $4.68(\mathrm{~d}, J=$ $11.5 \mathrm{~Hz}, 1 \mathrm{H}), 4.64-4.54(\mathrm{~m}, 5 \mathrm{H}), 4.44(\mathrm{ddd}, J=20.1 \mathrm{~Hz}, J=10.1 \mathrm{~Hz}, J=5.2 \mathrm{~Hz}, 1 \mathrm{H}), 4.15-$ $4.04(\mathrm{~m}, 4 \mathrm{H}), 3.94-3.82(\mathrm{~m}, 4 \mathrm{H}), 1.15(\mathrm{t}, J=7.2 \mathrm{~Hz}, 3 \mathrm{H}), 1.04(\mathrm{~s}, 9 \mathrm{H}) .{ }^{19} \mathrm{~F}$ NMR $(282.5 \mathrm{MHz}$, $\left.\mathrm{CDCl}_{3}\right) \delta-113.1(\mathrm{bd}, J=248.3 \mathrm{~Hz}, 1 \mathrm{~F}),-116.4(\mathrm{dd}, J=256.9 \mathrm{~Hz}, J=20.4 \mathrm{~Hz}, 1 \mathrm{~F}) .{ }^{13} \mathrm{C} \mathrm{NMR}$ $\left(75.5 \mathrm{MHz}, \mathrm{CDCl}_{3}\right) \delta 163.0(\mathrm{t}, J=29.7 \mathrm{~Hz}), 138.3,138.2,137.8,135.9,135.7,133.7,133.3$, $129.8,129.7,128.6,128.5,128.4,128.1,128.0,127.95,127.9,127.85,127.7,115.0(\mathrm{t}, J=$ 254.3 Hz), 77.2, 77.1, 73.8, 73.6, $72.8(\mathrm{dd}, J=28.9 \mathrm{~Hz}, J=22.7 \mathrm{~Hz}), 72.7,72.3$, 72.2, 63.0, 62.6, 26.9, 19.4, 13.9. IR (neat) $v_{\max }$ 3031, 2931, 2858, 1770. MS (ESI+) $: \mathrm{m} / \mathrm{z}=833.1$ $\left([\mathrm{M}+\mathrm{K}]^{+}\right), 817.1\left([\mathrm{M}+\mathrm{Na}]^{+}\right)$. Anal. Calcd. for $\mathrm{C}_{47} \mathrm{H}_{52} \mathrm{~F}_{2} \mathrm{O}_{7} \mathrm{Si}: \mathrm{C}, 71.01 ; \mathrm{H}, 6.59$. Found $\mathrm{C}$, $71.11 ; \mathrm{H}, 6.77$.

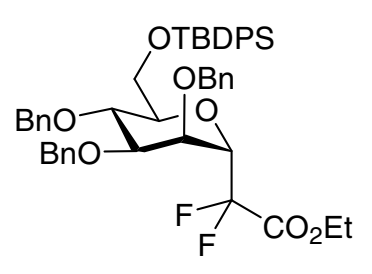

12

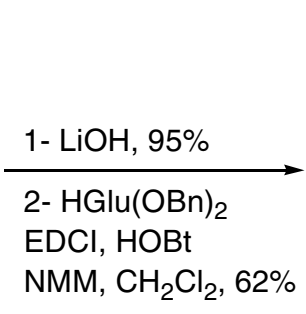

$\mathrm{NMM}, \mathrm{CH}_{2} \mathrm{Cl}_{2}, 62 \%$

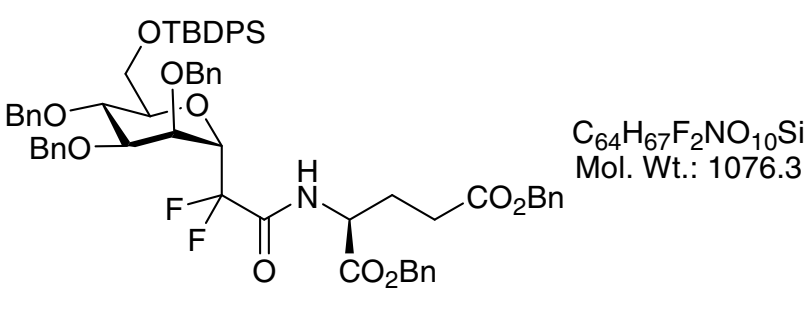

13 


\section{Di- $O$-benzyl $\quad N$-[2-(2,3,4-tri- $O$-benzyl-6- $O$-(tert-butyldiphenylsilyl)- $\alpha$-D-mannopyrano-}

syl)-2,2-difluoroacetyl]-L-glutamate (13). To a solution of 12 (231 mg, 0.29 mmol, 1 equiv) in THF ( $2 \mathrm{~mL})$ was added $\mathrm{LiOH}(14.3 \mathrm{mg}, 0.59 \mathrm{mmol}, 2$ equiv) dissolved in water $(1 \mathrm{~mL})$. The reaction was stirred at $\mathrm{rt}$, monitored by TLC and more $\mathrm{LiOH}$ in water was added if necessary. $\mathrm{CH}_{2} \mathrm{Cl}_{2}(10 \mathrm{~mL})$ was added and the reaction mixture was acidified to $\mathrm{pH} 1$ by addition of $\mathrm{HCl} 1 \mathrm{~N}$. The aqueous layer was extracted with $\mathrm{CH}_{2} \mathrm{Cl}_{2}(3 \times 5 \mathrm{~mL})$ and the collected organics were washed with water and evaporated under reduced pressure. The crude acid was obtained as a colourless oil (210 mg, $95 \%)$ after drying under vacuum and was used for the next step without further purification. This residue was dissolved in $\mathrm{CH}_{2} \mathrm{Cl}_{2}(2 \mathrm{~mL})$ and to this solution was added $\mathrm{HGlu}(\mathrm{OBn})_{2}$.TsOH (170 mg, $\left.0.34 \mathrm{mmol}\right)$, HOBt (49 mg, 0.29 mmol), NMM ( $80 \mu \mathrm{L}, 0.72 \mathrm{mmol})$ and EDC (70 mg, $0.36 \mathrm{mmol})$. The mixture was stirred for $36 \mathrm{~h}$ and then diluted with EtOAc $(10 \mathrm{~mL})$. The solution was washed with $10 \%$ aq. citric acid $(10 \mathrm{~mL})$, sat. $\mathrm{NaHCO}_{3}(10 \mathrm{~mL})$ and brine $(10 \mathrm{~mL})$. The organic layer was dried over $\mathrm{MgSO}_{4}$ and evaporated under reduced pressure. Purification by column chromatography (10\% EtOAc in cyclohexane) afforded 13 as colourless oil $(183 \mathrm{mg}, 62 \%) .[\alpha]_{\mathrm{D}}:+3.2\left(c 1.5, \mathrm{CHCl}_{3}\right) .{ }^{1} \mathrm{H}$ NMR (300 MHz, $\left.\mathrm{CDCl}_{3}\right) \delta$ 7.66-7.61 (m, 4H), 7.42-7.12 (m, 31H), $7.04(\mathrm{~d}, J=7.6 \mathrm{~Hz}, 1 \mathrm{H})$, 5.04 (s, 2H), 4.99 (d, $J=12.2 \mathrm{~Hz}, 1 \mathrm{H}), 4.89$ (d, $J=12.2 \mathrm{~Hz}, 1 \mathrm{H}), 4.79-4.44$ (m, 7H), 4.13$4.06(\mathrm{~m}, 2 \mathrm{H}), 3.95-3.70(\mathrm{~m}, 4 \mathrm{H}), 2.40-2.12(\mathrm{~m}, 3 \mathrm{H}), 2.01-1.89(\mathrm{~m}, 1 \mathrm{H}), 0.99(\mathrm{~s}, 9 \mathrm{H}) .{ }^{19} \mathrm{~F}$ NMR $\left(282.5 \mathrm{MHz}, \mathrm{CDCl}_{3}\right) \delta-110.6(\mathrm{~d}, J=258.6 \mathrm{~Hz}, 1 \mathrm{~F}),-117.0(\mathrm{dd}, J=258.6 \mathrm{~Hz}, J=20.4$ $\mathrm{Hz}, 1 \mathrm{~F}) .{ }^{13} \mathrm{C} \mathrm{NMR}\left(75.5 \mathrm{MHz}, \mathrm{CDCl}_{3}\right) \delta 172.5,170.5,162.9(\mathrm{t}, J=27.0 \mathrm{~Hz}), 138.5,138.3$, 138.0, 136.0, 135.7, 135.6, 135.0, 133.8, 133.3, 129.7, 129.0, 128.8-127.7 $\left(\mathrm{CH}_{\mathrm{Ar}}\right), 116.2(\mathrm{t}, J$ $=258.2 \mathrm{~Hz}), 77.7,77.4,73.8,73.7,72.8(\mathrm{t}, J=22.0 \mathrm{~Hz}), 72.6,72.2,72.1,67.6,66.7,62.3$, 51.8, 29.9, 27.1, 26.9, 19.4. IR (neat) $v_{\max } 3351,3032,2930,2857,1738,1710 \mathrm{~cm}^{-1}$. MS $(\mathrm{ESI}+): \mathrm{m} / \mathrm{z}=1114.4\left([\mathrm{M}+\mathrm{K}]^{+}\right), 1098.5\left([\mathrm{M}+\mathrm{Na}]^{+}\right)$. Anal. Calcd. for $\mathrm{C}_{64} \mathrm{H}_{67} \mathrm{~F}_{2} \mathrm{NO}_{10} \mathrm{Si}: \mathrm{C}$, 71.42; H, 6.27; N, 1.30. Found C, 71.34; H, 6.31; N, 1.34.

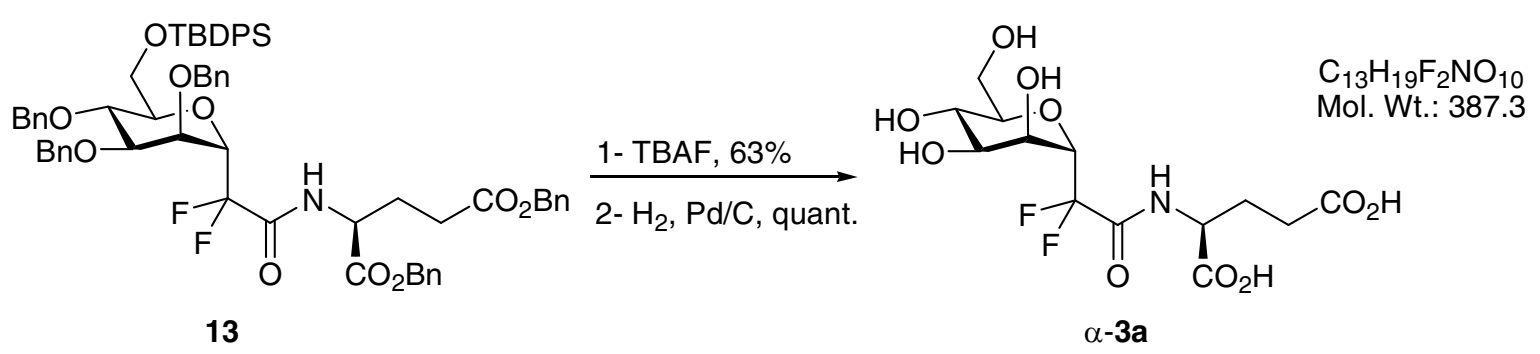

$N$-(2,2-Difluoro-2-( $\alpha$-D-mannopyranosyl)acetyl)-L-glutamic acid ( $\alpha$-3a). To a solution of 13 (92 mg, $0.085 \mathrm{mmol})$ in THF ( $3 \mathrm{~mL}$ ) was added TBAF (1M solution in THF, $0.1 \mathrm{~mL}, 0.1$ 
mmol). The mixture was stirred at $\mathrm{rt}$ for $3 \mathrm{~h}$ then quenched with sat. $\mathrm{NH}_{4} \mathrm{Cl}$. The aqueous layer was extracted with EtOAc $(3 \times 5 \mathrm{~mL})$ and the collected organics were washed with brine ( $5 \mathrm{~mL}$ ), dried over $\mathrm{MgSO}_{4}$ and evaporated under reduced pressure. Purification by column chromatography (20\% EtOAc in cyclohexane) afforded the desired deprotected glycopeptide as a colorless oil $(45 \mathrm{mg}, 63 \%) .{ }^{1} \mathrm{H}$ NMR $\left(300 \mathrm{MHz}, \mathrm{CDCl}_{3}\right) \delta 7.62(\mathrm{bd}, J=6.2 \mathrm{~Hz}, 1 \mathrm{H})$, 7.29-7.04 (m, 25H), 5.14-4.94 (m, 4H), 4.61-4.35 (m, 8H), 4.08-4.02 (m, 1H), 3.90-3.66 (m, $3 \mathrm{H}), 3.56-3.50(\mathrm{~m}, 2 \mathrm{H}), 2.96(\mathrm{bs}, 1 \mathrm{H}), 2.39-2.32(\mathrm{~m}, 2 \mathrm{H}), 2.22-2.11(\mathrm{~m}, 1 \mathrm{H}), 2.07-1.91$ (m, 1H). ${ }^{19} \mathrm{~F}$ NMR $\left(282.5 \mathrm{MHz}, \mathrm{CDCl}_{3}\right) \delta-108.8(\mathrm{~d}, J=259.4 \mathrm{~Hz}, 1 \mathrm{~F}),-119.8(\mathrm{dd}, J=259.4 \mathrm{~Hz}$, $J=25.3 \mathrm{~Hz}, 1 \mathrm{~F}) .{ }^{13} \mathrm{C} \mathrm{NMR}\left(75.5 \mathrm{MHz}, \mathrm{CDCl}_{3}\right) \delta 173.3,171.0,163.5(\mathrm{t}, J=29.3 \mathrm{~Hz}), 138.1$, 138.0, 137.6, 135.5, 134.9, 128.8, 128.75, 128.7, 128.5, 128.4, 128.3, 128.0, 127.9, 116.3 (t, $J$ = 264.5 Hz), 77.9, 77.4, 74.8, 73.8, 72.8, 72.6, $72.5(\mathrm{t}, J=22.8 \mathrm{~Hz}), 71.8,67.9,67.1,62.3$, $52.6,30.2,25.6$.

To a solution of this compound (45 mg, $0.054 \mathrm{mmol})$ in THF (2 mL) was added water $(1 \mathrm{~mL})$, few drops of conc. $\mathrm{HCl}$ and $\mathrm{Pd} / \mathrm{C}$ (tip of spatula). The reaction mixture was purged with $\mathrm{H}_{2}$ and stirred under an $\mathrm{H}_{2}$ atmosphere for $24 \mathrm{~h}$. The suspension was then filtered, washed with water and the filtrate was evaporated under reduced pressure. The residue was dried by successive co-evaporations with toluene then redissolved in water, filtered and lyophilised to afford $\alpha-3 \mathbf{a}\left(20 \mathrm{mg}\right.$, quant.) as a white solid; $\mathrm{mp} 118-120^{\circ} \mathrm{C}$; $[\alpha]_{\mathrm{D}}:+15.6\left(c 0.6, \mathrm{H}_{2} \mathrm{O}, \lambda=436\right.$ nm). ${ }^{1} \mathrm{H}$ NMR $\left(300 \mathrm{MHz}, \mathrm{D}_{2} \mathrm{O}\right) \delta 4.38(\mathrm{ddd}, J=21.9 \mathrm{~Hz}, J=11.8 \mathrm{~Hz}, J=2.4 \mathrm{~Hz}, 1 \mathrm{H}), 4.25(\mathrm{t}$, $J=2.7 \mathrm{~Hz}, 1 \mathrm{H}), 4.16(\mathrm{dd}, J=4.6 \mathrm{~Hz}, J=8.8 \mathrm{~Hz}, 1 \mathrm{H}), 3.91-3.86(\mathrm{~m}, 1 \mathrm{H}), 3.79-3.70(\mathrm{~m}, 4 \mathrm{H})$, 2.23 (app t, $J=7.7 \mathrm{~Hz}, 2 \mathrm{H}), 2.14-2.02(\mathrm{~m}, 1 \mathrm{H}), 1.99-1.86(\mathrm{~m}, 1 \mathrm{H}) .{ }^{19} \mathrm{~F}$ NMR $(282.5 \mathrm{MHz}$, $\left.\mathrm{D}_{2} \mathrm{O}\right) \delta-110.2(\mathrm{dd}, J=259.1 \mathrm{~Hz}, J=11.8 \mathrm{~Hz}, 1 \mathrm{~F}),-114.5(\mathrm{dd}, J=259.1 \mathrm{~Hz}, J=21.5 \mathrm{~Hz}, 1 \mathrm{~F})$. ${ }^{13} \mathrm{C}$ NMR $\left(75.5 \mathrm{MHz}, \mathrm{D}_{2} \mathrm{O}\right) \delta 182.5,177.9,164.2(\mathrm{t}, J=27.9 \mathrm{~Hz}), 116.4(\mathrm{t}, J=259.3 \mathrm{~Hz})$, $78.2,76.8(\mathrm{dd}, J=28.5 \mathrm{~Hz}, J=22.2 \mathrm{~Hz}), 71.3,66.7,66.6,61.2,56.1,34.2,28.0$. MS (ESI-) : $\mathrm{m} / \mathrm{z}=386.0\left([\mathrm{M}-\mathrm{H}]^{-}\right)$. HRMS (TOF-) calcd for $\mathrm{C}_{13} \mathrm{H}_{18} \mathrm{~F}_{2} \mathrm{NO}_{10} \mathrm{~m} / \mathrm{z}(\mathrm{M}-\mathrm{H})^{-}$386.0898, found 386.0882 .

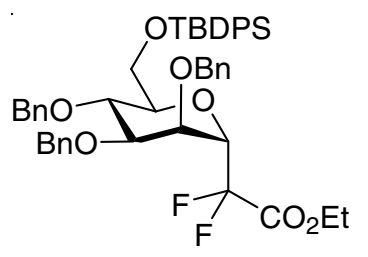

12

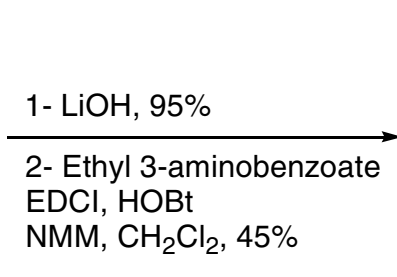
EDCl, $\mathrm{HOBt}, \mathrm{CH}_{2} \mathrm{Cl}_{2}, 45 \%$

Ethyl $\quad N$-[2-(2,3,4-tri- $O$-benzyl-6- $O$-(tert-butyldiphenylsilyl)- $\alpha$-D-mannopyranosyl)-2,2difluoroacetyl]-3-aminobenzoate (14). To a solution of 12 (150 mg, 0.19 mmol, 1 equiv) in 
THF (2 mL) was added $\mathrm{LiOH}(9.1 \mathrm{mg}, 0.38 \mathrm{mmol}, 2$ equiv) dissolved in water $(1 \mathrm{~mL})$. The reaction was stirred at rt, monitored by TLC and more LiOH in water was added if necessary. $\mathrm{CH}_{2} \mathrm{Cl}_{2}(10 \mathrm{~mL})$ was added and the reaction mixture was acidified to $\mathrm{pH} 1$ by addition of $\mathrm{HCl}$ $1 \mathrm{~N}$. The aqueous layer was extracted with $\mathrm{CH}_{2} \mathrm{Cl}_{2}(3 \times 5 \mathrm{~mL})$ and the collected organics were washed with water and evaporated under reduced pressure. The crude acid was obtained as a colourless oil (138 mg, $95 \%$ ) after drying under vacuum and was used for the next step without further purification. This residue was dissolved in $\mathrm{CH}_{2} \mathrm{Cl}_{2}(2 \mathrm{~mL})$ and to this solution was added ethyl 3-aminobenzoate (48 mg, $0.29 \mathrm{mmol}$ ), HOBt (39 mg, $0.29 \mathrm{mmol}$ ), NMM (32 $\mu \mathrm{L}, 0.29 \mathrm{mmol})$ and $\mathrm{EDC}(56 \mathrm{mg}, 0.29 \mathrm{mmol})$. The mixture was stirred for $36 \mathrm{~h}$ and then diluted with EtOAc $(10 \mathrm{~mL})$. The solution was washed with $10 \%$ aq. citric acid $(10 \mathrm{~mL})$, sat. $\mathrm{NaHCO}_{3}(10 \mathrm{~mL})$ and brine $(10 \mathrm{~mL})$. The organic layer was dried over $\mathrm{MgSO}_{4}$ and evaporated under reduced pressure. Purification by column chromatography (10\% EtOAc in cyclohexane) afforded 14 as colourless oil $(73 \mathrm{mg}, 45 \%)$. [ $\alpha]_{\mathrm{D}}:+12.9\left(c 0.34, \mathrm{CHCl}_{3}\right) .{ }^{1} \mathrm{H}$ NMR (300 MHz, $\left.\mathrm{CDCl}_{3}\right) \delta 8.13$ (bs, 1H), 8.01 (bs, 1H), 7.85-7.78 (m, 2H), 7.65-7.61 (m, $4 \mathrm{H}), 7.40-7.10(\mathrm{~m}, 22 \mathrm{H}), 4.63-4.46(\mathrm{~m}, 7 \mathrm{H}), 4.37$ (q, $J=7.1 \mathrm{~Hz}, 2 \mathrm{H}), 4.18-4.13(\mathrm{dd}, J=6.2$ $\mathrm{Hz}, J=2.7 \mathrm{~Hz}, 1 \mathrm{H}), 4.00-3.85(\mathrm{~m}, 5 \mathrm{H}), 1.39(\mathrm{t}, J=7.1 \mathrm{~Hz}, 3 \mathrm{H}), 0.99(\mathrm{~s}, 9 \mathrm{H}) .{ }^{19} \mathrm{~F} \mathrm{NMR}$ $\left(282.5 \mathrm{MHz}, \mathrm{CDCl}_{3}\right) \delta-112.5(\mathrm{~d}, J=254.8 \mathrm{~Hz}, 1 \mathrm{~F}),-117.1(\mathrm{dd}, J=259.4 \mathrm{~Hz}, J=17.2 \mathrm{~Hz}$, 1F). ${ }^{13} \mathrm{C} \mathrm{NMR}\left(75.5 \mathrm{MHz}, \mathrm{CDCl}_{3}\right) \delta 166.0,161.1(\mathrm{t}, J=27.6 \mathrm{~Hz}), 138.0,137.6,136.4,135.7$, $135.6,133.4$, 133.2, 131.4, 129.8, 129.7, 129.3, 129.0, 128.5, 128.45, 128.4, 128.1, 128.0, $127.9,127.85,127.8,127.7,126.4,124.7,121.2,115.9$ (t, $J=258.7 \mathrm{~Hz}), 78.0,75.9,74.1$, 73.0, 72.7, 72.2, 72.1, $71.8(\mathrm{dd}, J=27.4 \mathrm{~Hz}, J=23.6 \mathrm{~Hz}), 62.5,61.3,26.8,19.2$, 14.4. MS $(\mathrm{ESI}+): \mathrm{m} / \mathrm{z}=952.4\left([\mathrm{M}+\mathrm{K}]^{+}\right), 936.4\left([\mathrm{M}+\mathrm{Na}]^{+}\right) . \quad \mathrm{HRMS} \quad(\mathrm{TOF}+)$ calcd for $\mathrm{C}_{54} \mathrm{H}_{57} \mathrm{~F}_{2} \mathrm{NO}_{8} \mathrm{SiNa} m / z(\mathrm{M}+\mathrm{Na})^{+}$936.3719, found 936.3712 .

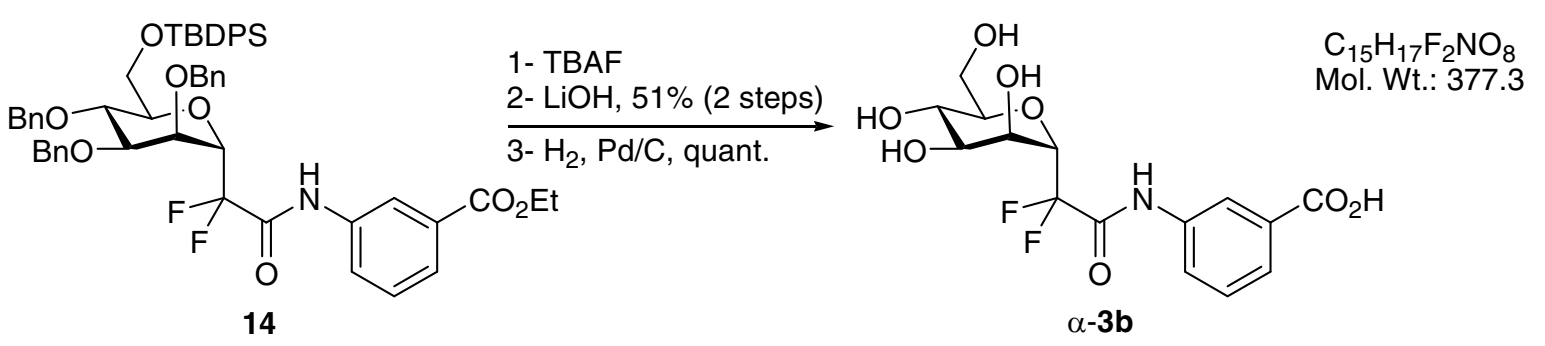

$N$-[2,2-Difluoro-2-( $\alpha$-D-mannopyranosyl)acetyl]-3-aminobenzoic acid $(\alpha-3 b)$. To a solution of 14 (74 mg, $0.08 \mathrm{mmol})$ in THF (3 mL) was added TBAF (1M solution in THF, $0.15 \mathrm{~mL}, 0.15 \mathrm{mmol}$ ). The mixture was stirred at $\mathrm{rt}$ for $3 \mathrm{~h}$ then quenched with sat. $\mathrm{NH}_{4} \mathrm{Cl}$. The aqueous layer was extracted with EtOAc $(3 \times 5 \mathrm{~mL})$ and the collected organics were washed with brine $(5 \mathrm{~mL})$, dried over $\mathrm{MgSO}_{4}$ and evaporated under reduced pressure. The 
crude mixture was dissolved in THF $(2 \mathrm{~mL})$ and $\mathrm{LiOH}$ (4.0 $\mathrm{mg}, 0.16 \mathrm{mmol}, 2$ equiv) dissolved in water $(1 \mathrm{~mL})$ was added. The mixture was stirred at $\mathrm{rt}$ and monitored by TLC (40\% EtOAc in cyclohexane): two more additions of $\mathrm{LiOH}(4.0 \mathrm{mg}, 0.16 \mathrm{mmol}, 2$ equiv) and $36 \mathrm{~h}$ of reaction were usually required for a complete conversion of the starting material. The reaction mixture was then acidified to $\mathrm{pH} 1$ by addition of $\mathrm{HCl} 1 \mathrm{~N}$. The aqueous layer was extracted with EtOAc $(3 \times 5 \mathrm{~mL})$ and the collected organics were washed with brine, dried over $\mathrm{MgSO}_{4}$ and evaporated under reduced pressure. Purification by column chromatography (1\% AcOH and 30\% EtOAc in cyclohexane) afforded the desired benzylated acid as colourless oil $(26 \mathrm{mg}, 51 \%) . \mathrm{Rf}=0.15(1 \% \mathrm{AcOH}$ and $40 \%$ EtOAc in cyclohexane $) .{ }^{1} \mathrm{H}$ NMR $\left(300 \mathrm{MHz}, \mathrm{CDCl}_{3}\right) \delta 8.65$ (bs, 1H), 8.06-8.00 (m, 2H), $7.80(\mathrm{~d}, J=6.2 \mathrm{~Hz}, 1 \mathrm{H}), 7.41-7.13$ $(\mathrm{m}, 16 \mathrm{H}), 4.75-4.45(\mathrm{~m}, 7 \mathrm{H}), 4.19-4.15(\mathrm{~m}, 1 \mathrm{H}), 4.03-3.82(\mathrm{~m}, 4 \mathrm{H}), 3.66-3.57(\mathrm{~m}, 2 \mathrm{H}) .{ }^{19} \mathrm{~F}$ $\operatorname{NMR}\left(282.5 \mathrm{MHz}, \mathrm{CDCl}_{3}\right) \delta-109.9(\mathrm{~d}, J=261.7 \mathrm{~Hz}, 1 \mathrm{~F}),-118.2\left(\operatorname{app~d}, J_{\text {app }}=244.5 \mathrm{~Hz}, 1 \mathrm{~F}\right)$. ${ }^{13} \mathrm{C}$ NMR $\left(75.5 \mathrm{MHz}, \mathrm{CDCl}_{3}\right) \delta 170.0,161.5(\mathrm{t}, J=27.6 \mathrm{~Hz}), 137.8,137.6,137.4,136.6$, $130.5,129.5,129.0,128.7,128.6,128.5,128.3,128.2,128.1,128.0,127.1,125.5,122.0$, $116.2(\mathrm{dd}, J=263.3 \mathrm{~Hz}, J=257.6 \mathrm{~Hz}), 77.5,76.3,74.8,73.4,73.0,72.6,72.0,71.4(\mathrm{dd}, J=$ $30.5 \mathrm{~Hz}, J=21.9 \mathrm{~Hz}), 61.4$.

To a solution of this compound (26 mg, $0.04 \mathrm{mmol})$ in THF $(2 \mathrm{~mL})$ was added water $(1 \mathrm{~mL})$, few drops of conc. $\mathrm{HCl}$ and $\mathrm{Pd} / \mathrm{C}$ (tip of spatula). The reaction mixture was purged with $\mathrm{H}_{2}$ and stirred under an $\mathrm{H}_{2}$ atmosphere for $24 \mathrm{~h}$. The suspension was then filtered, washed with water and the filtrate was evaporated under reduced pressure. The residue was dried by successive co-evaporations with toluene then redissolved in water, filtered and lyophilised to afford $\alpha-3 b$ (15 mg, quant.) as a yellowish solid; mp 110-112 ${ }^{\circ} \mathrm{C} ;[\alpha]_{\mathrm{D}}:+30.5\left(c 0.8, \mathrm{H}_{2} \mathrm{O}\right) .{ }^{1} \mathrm{H}$ $\operatorname{NMR}\left(300 \mathrm{MHz}, \mathrm{D}_{2} \mathrm{O}\right) \delta 8.02(\mathrm{bs}, 1 \mathrm{H}), 7.85(\mathrm{~d}, J=7.7 \mathrm{~Hz}, 1 \mathrm{H}), 7.72-7.68(\mathrm{~m}, 1 \mathrm{H}), 7.50(\mathrm{t}, J$ $=8.0 \mathrm{~Hz}, 1 \mathrm{H}), 4.48(\mathrm{ddd}, J=24.5 \mathrm{~Hz}, J=9.0 \mathrm{~Hz}, J=2.3 \mathrm{~Hz}, 1 \mathrm{H}), 4.34\left(\right.$ app t,$J_{\text {app }}=3.0 \mathrm{~Hz}$, $1 \mathrm{H}), 3.94-3.90(\mathrm{~m}, 1 \mathrm{H}), 3.79-3.67(\mathrm{~m}, 4 \mathrm{H}).) .{ }^{19} \mathrm{~F} \mathrm{NMR}\left(282.5 \mathrm{MHz}, \mathrm{D}_{2} \mathrm{O}\right) \delta-109.8(\mathrm{dd}, J=$ $258.2 \mathrm{~Hz}, J=9.2 \mathrm{~Hz}, 1 \mathrm{~F}),-115.7(\mathrm{dd}, J=258.2 \mathrm{~Hz}, J=24.1 \mathrm{~Hz}, 1 \mathrm{~F}) .{ }^{13} \mathrm{C}$ NMR $(75.5 \mathrm{MHz}$, $\left.\mathrm{D}_{2} \mathrm{O}\right) \delta 169.9,163.3(\mathrm{t}, J=29.3 \mathrm{~Hz}), 135.7,130.9,129.9,128.0,127.8,123.8,116.4(\mathrm{dd}, J=$ $263.9 \mathrm{~Hz}, J=258.2 \mathrm{~Hz}), 78.4,76.6(\mathrm{dd}, J=29.9 \mathrm{~Hz}, J=22.4 \mathrm{~Hz}), 71.3,66.8,66.3,61.1$. MS $(\mathrm{ESI}-): \mathrm{m} / \mathrm{z}=376.1\left([\mathrm{M}-\mathrm{H}]^{-}\right)$. HRMS $(\mathrm{TOF}-)$ calcd for $\mathrm{C}_{15} \mathrm{H}_{16} \mathrm{~F}_{2} \mathrm{NO}_{8} \mathrm{~m} / z(\mathrm{M}-\mathrm{H})^{-}$ 376.0843 , found 376.0842 . 